Check for updates

Cite this: RSC Adv., 2017, 7, 54823

Received 29th September 2017 Accepted 26th November 2017

DOI: $10.1039 / c 7 r a 10788 a$

rsc.li/rsc-advances

\section{Preparation of conductive composite hydrogels from carboxymethyl cellulose and polyaniline with a nontoxic crosslinking agent}

\author{
Junrong Li, ${ }^{a}$ Liangjing Fang, ${ }^{a}$ William R. Tait, ${ }^{\mathrm{b}}$ Luyi Sun, ${ }^{\mathrm{b}}{ }^{\mathrm{b}}$ Lihong Zhao $^{\mathrm{a}}$ \\ and Liying Qian (D)*a
}

Conductive composite hydrogels based on sodium carboxymethyl cellulose (CMC) and polyaniline (PAn) were prepared via the semi-interpenetrating polymer network (semi-IPN) method, using glycerol diglycidyl ether (GDE) as the crosslinking agent. The structures of the resulting composite hydrogels were characterized by ${ }^{13} \mathrm{C}-\mathrm{NMR}, \mathrm{FT}-\mathrm{IR}$, and SEM. The composite hydrogels with various concentrations of CMC presented a similar swelling kinetic behaviour and a relatively high swelling ratio. With increasing CMC concentration, both the compressive fracture stress and modulus of the composite hydrogels improved gradually. Though the modulus of the composite hydrogels increased with more GDE added, the compressive fracture stress decreased when the crosslinking density was too high. The electrical conductivity of the composite hydrogels increased first and then decreased slightly with increasing dosages of CMC and GDE. Doping with aromatic sulfonate is a good approach to improve the conductivity of the composite hydrogels and the conductivity reached $6.31 \times 10^{-3} \mathrm{~S} \mathrm{~cm}^{-1}$ after doping with sodium benzene sulfonate (BSNa).

\section{Introduction}

Conductive composite hydrogels consisting of hydrogels and conductive polymers (CPs) have received considerable attention for their diversified applications in biosensors, fuel cells, biomedical technology, pharmaceuticals, environmental technology, tissue engineering, and so on. ${ }^{\mathbf{1 - 4}}$ For practical applications and to meet the requirements for the fabrication of flexible devices, these materials should demonstrate good conductivity and mechanical properties. ${ }^{5}$

Hydrogels are usually obtained from natural polymers, such as cellulose ${ }^{6}$ and its derivatives, ${ }^{7}$ chitosan $^{8}$ and sodium alginate, ${ }^{9}$ by physical $^{10}$ or chemical ${ }^{\mathbf{1 1}}$ crosslinking methods. As anion linear cellulose ether, the introduction of carboxymethyl and hydroxyl groups on the cellulose makes it highly soluble in water and chemically reactive, ${ }^{\mathbf{1 2}}$ therefore, CMC is commonly used as hydrogel substrate due to its biocompatibility, biodegradability, lack of taste, and nontoxicity. ${ }^{13}$ Composite hydrogels based on CMC have been widely used in many applications; for example, CMC/hydroxyapatite hydrogel (1,3-diaminopropane as the crosslinking agent) was used for seeding human dental pulp stem cells, ${ }^{\mathbf{1 4}}$ CMC-g-PAA hydrogel

${ }^{a}$ State Key Laboratory of Pulp and Paper Engineering, South China University of Technology, Guangzhou, Guangdong 510641, P. R. China. E-mail: lyqian@scut.edu. cn; Fax: +86-020-87111770; Tel: +86-020-87111770

${ }^{b}$ Department of Chemical \& Biomolecular Engineering and Polymer Program, Institute of Materials Science, University of Connecticut, Storrs, Connecticut 06269, USA
( $N, N^{\prime}$-methylenebisacrylamide as the crosslinking agent) was used for drug-controlled delivery systems, ${ }^{15}$ CMC- $g$-PDMAEMA hydrogel (ethylene glycol dimethacrylate as the crosslinking agent) was used for the removal of synthetic dyes in wastewater, ${ }^{\mathbf{1 6}}$ and $\mathrm{CMC} / \mathrm{ZnO}$ nanoparticles hydrogel (epichlorohydrin as the crosslinking agent) was used as antibacterial material. ${ }^{17}$ Compared with chemically crosslinked hydrogels, the stabilities of the hydrogels prepared by physical crosslinking are relatively weak. ${ }^{18}$ In general, a crosslinking agent is necessary for chemical crosslinking reactions, which makes crosslinking costly. In addition, the toxicity of residual crosslinking agents often limits the application in biomedical materials. Therefore, utilization of nontoxic crosslinking agents with high reaction efficiency is becoming important for chemically crosslinked hydrogels, especially for some applications in biomedical and tissue engineering.

Polyaniline (PAn) has good biocompatibility, ${ }^{19}$ environmental stability, high electrical conductivity, and has many potential applications in various fields. ${ }^{\mathbf{2 0 , 2 1}}$ There are many reports about the applications of composite hydrogels containing PAn, such as regenerative processes in nerve cells, ${ }^{22}$ antibacterial materials, ${ }^{23}$ supercapacitors ${ }^{24}$ and adsorption materials. ${ }^{25}$ However, there is limited literature on conductive composite hydrogels from combined CMC and PAn.

Herein, this study aimed to design a conductive composite hydrogel composed of NaCMC and PAn, which is expected to be used in biomedical materials. As the conductive component, PAn was interpenetrated in a NaCMC matrix using high 
efficiency and nontoxic glycerol diglycidyl ether (GDE) as the crosslinking agent. The structure and morphology of the obtained hydrogels were characterized by ${ }^{13} \mathrm{C}-\mathrm{NMR}$, FT-IR, and SEM. The thermal stability, swelling, mechanical properties, and conductivity of the composite hydrogels were investigated as well.

\section{Results and discussion}

\section{Structure and morphology analysis}

Solid-state NMR spectra of CMC and CMC hydrogels are shown in Fig. 1. Absorption peaks of six carbon atoms in the polysaccharide unit were in the range of $50-100 \mathrm{ppm} .{ }^{26}$ The peaks at 103.48, 80.91, and $61.47 \mathrm{ppm}$ were the absorption of $\mathrm{C} 1, \mathrm{C} 4$, and C6, respectively. The peaks between 71 and $80 \mathrm{ppm}$ belonged to C2, C3, and C5. The peak at $177.08 \mathrm{ppm}$ was specific to the chemical shift of $-\mathrm{COO}^{-}$. The new peak that appeared at $32.5 \mathrm{ppm}$ in the $\mathrm{CMC}$ hydrogel was caused by the $-\mathrm{CH}_{2}-$ of GDE. The results demonstrated that GDE reacted with the $-\mathrm{OH}$ group of CMC and formed a network structure.

FT-IR spectra of pristine PAn, CMC hydrogels, and CMC/PAn hydrogels are shown in Fig. 2. As seen in Fig. 2(a), $3434 \mathrm{~cm}^{-1}$ was the anti-symmetric and symmetric stretching vibration absorption peak of $-\mathrm{NH}_{2}$, and the characteristic peak at $1291 \mathrm{~cm}^{-1}$ was attributed to the stretching vibration of $\mathrm{C}-\mathrm{N}$. The peak at $3434 \mathrm{~cm}^{-1}$ in Fig. 2(b) was ascribed to the stretching vibration absorption of $-\mathrm{OH}$ in CMC. Peaks at 1619 and $1419 \mathrm{~cm}^{-1}$ were caused by the carboxylic sodium in CMC. In Fig. 2(c), the specific absorption peak of PAn was presented at $1291 \mathrm{~cm}^{-1}$. Carboxylate was turned into carboxylic acid during the preparation of the composite hydrogels, so the absorption peak of $-\mathrm{COOH}\left(1721 \mathrm{~cm}^{-1}\right)$ also appeared on Fig. 2(c). As a result of the hydrogen-bond interactions between - $\mathrm{NH}-$ of PAn and $-\mathrm{OH}$ of $\mathrm{CMC}$, the absorption peaks of the two groups shifted toward a lower wavenumber $\left(3222 \mathrm{~cm}^{-1}\right)$. The peak at $1063 \mathrm{~cm}^{-1}$ was due to the stretching vibration of $\mathrm{C}-\mathrm{O}$ of the secondary alcohol in GDE.

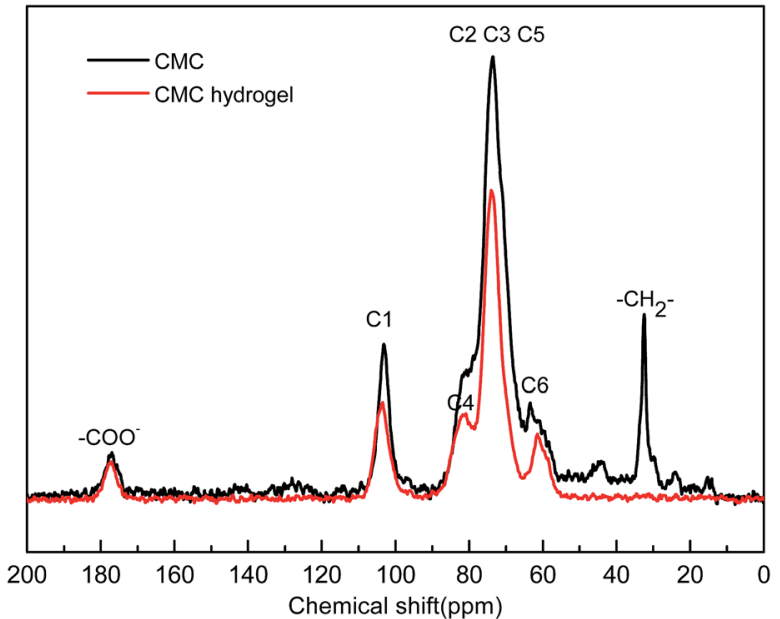

Fig. 1 Solid-state ${ }^{13} \mathrm{C}-\mathrm{NMR}$ spectra of $\mathrm{CMC}$ and $\mathrm{CMC}$ hydrogel $(7 \%$ CMC, $n_{\mathrm{GDE}}: n_{\mathrm{AGU}}=1: 2$ ).

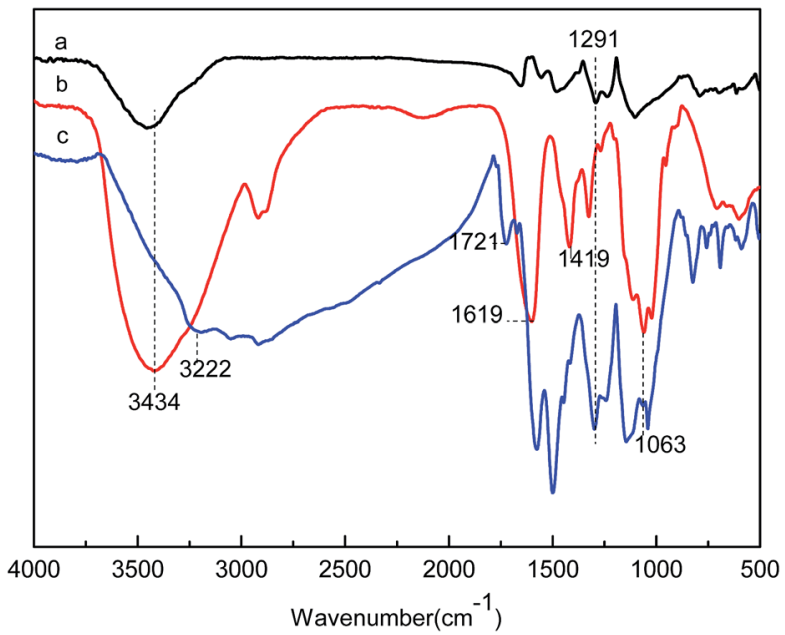

Fig. 2 FT-IR spectra of PAn (a), CMC hydrogels (b) and CMC/PAn composite hydrogels (c) $\left(7 \% \mathrm{CMC}, n_{\mathrm{GDE}}: n_{\mathrm{AGU}}=1: 2\right)$.

Fig. 3 shows the micromorphology of CMC hydrogels (a) and CMC/PAn hydrogels (b). Some interconnected voids with a dimension of 16-33 $\mu \mathrm{m}$ in CMC hydrogels were clearly visible, which means the hydrogel matrix had a high absorbing performance. These pore spaces were filled with PAn in the composite hydrogels, and this might have enhanced the mechanical properties of the material.

\section{Swelling property}

The swelling properties of hydrogels play an important role in their applications. The graphs of swelling ratio versus time of the CMC/PAn composite hydrogels are shown in Fig. 4. The composite hydrogels presented similar swelling kinetics even though their CMC concentrations were different. The weight of composite hydrogels increased dramatically in the first $35 \mathrm{~min}$; then the weights increased slowly until equilibrium was reached after 400 minutes. The swelling ratio of composite hydrogels decreased with increasing CMC because more polymer chains in solution lead to increased crosslinkage and denser hydrogel matrix which decreased the absorption of water. Meanwhile, PAn was easier to aggregate in a relatively compact network structure; the hydrogen bonds formed between $-\mathrm{OH}$ and $-\mathrm{NH}_{2}$ decreased the hydrophilicity of the composite hydrogels. It also could be seen from Fig. 4 that the swelling ratios of the composite hydrogels in this work were

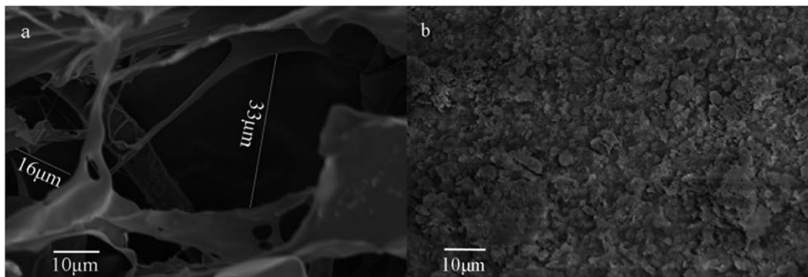

Fig. 3 SEM images of CMC hydrogels (a) and CMC/PAn composite hydrogels (b) $\left(7 \% \mathrm{CMC}, n_{\mathrm{GDE}}: n_{\mathrm{AGU}}=1: 2\right)$. 


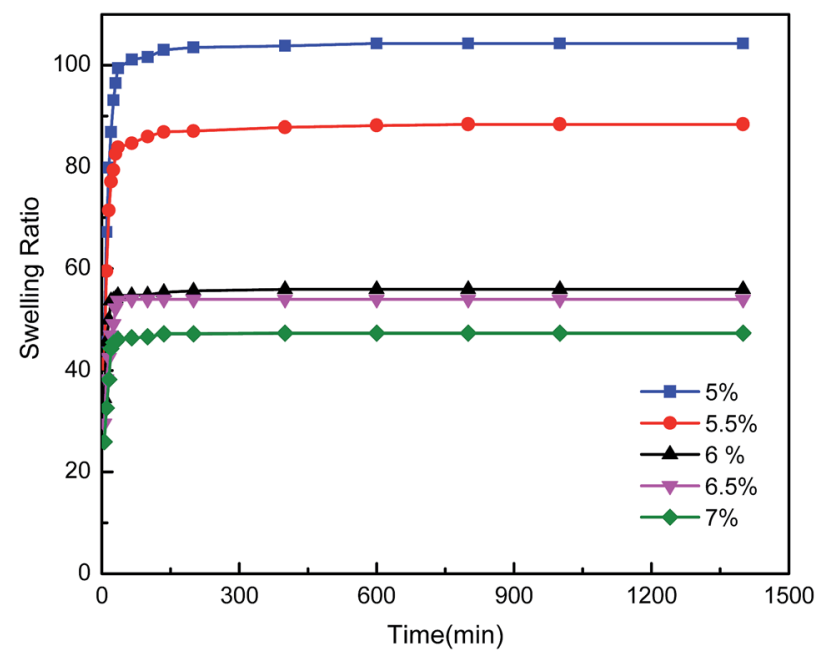

Fig. 4 Swelling kinetics of CMC/PAn composite hydrogels $\left(n_{\mathrm{GDE}}: n_{\mathrm{AGU}}=1: 2\right)$.

about 47-104, which are much higher than the swelling ratios of 5.5-10.6 of the CMCS/PAn hydrogels reported by Zhang. ${ }^{27}$

\section{Effects of CMC concentration on mechanical property and electrical conductivity}

The stress-strain curves of CMC/PAn hydrogels with different concentrations of CMC are shown in Fig. 5. The compressive fracture stress of the composite hydrogels increased as the CMC content increased. The composite gels showed better mechanical properties when the CMC concentrations were above $6 \%$ with the biggest compression fracture strength of $37.5 \mathrm{kPa}$ at $14.4 \%$ strain for $7 \%$ CMC concentration.

Modulus is also used for evaluation of the strength of the composite hydrogels and modulus of the hydrogel could be modified by changing the crosslinking time, monomer and crosslinker concentrations. Therefore, the modulus of the CMC/ PAn composite hydrogels were influenced by both the CMC

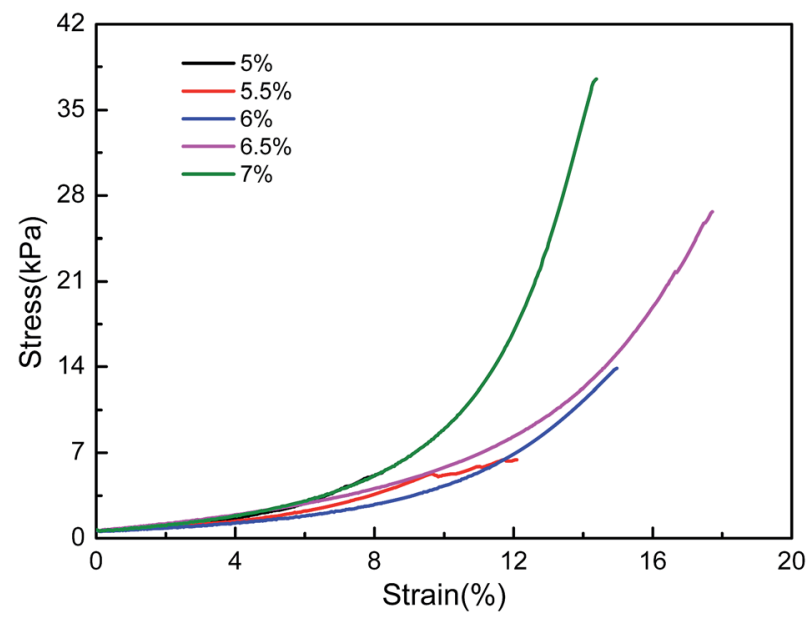

Fig. 5 Compressive stress-strain curves of CMC/PAn composite hydrogels with different concentrations of CMC $\left(n_{\mathrm{GDE}}: n_{\mathrm{AGU}}=1: 2\right)$. concentration and GDE dosage. The increasing of the CMC concentrations resulted in higher modulus of composite gels (Table 1) because more polymer chains in solution lead to a larger number of functional crosslinks. ${ }^{28}$ Especially, the modulus when CMC concentrations above $6 \%$ were almost two times of those less than or equal to $6 \%$ which means the crosslink density in hydrogel networks was relatively high. Therefore, the crosslinking degree of the composite hydrogels increased with increasing content of CMC, and so the strength of the hydrogels increased.

The electrical conductivities of the CMC/PAn hydrogels are listed in Table 1. The composite hydrogels got the highest conductivity, up to $7.73 \times 10^{-4} \mathrm{~S} \mathrm{~cm}^{-1}$, when the CMC concentration was $6 \%$, and then it decreased as concentration continued to increase. When CMC concentration was low in the reaction process, the crosslinking reaction was difficult to carry on; therefore the crosslinking degree of hydrogels was insufficient, and it was difficult to form the gel network. As a result, it was hard for PAn to be absorbed into the network to form a good electric channel, therefore the conductivity of composite gel was low. However, the structure of composite hydrogels became so condensed when the CMC concentration was higher than $6 \%$ that the free spaces for PAn absorption was decreased which resulted in a little decreased conductivity of the composite gels. This can be seen from the swelling ratio at various CMC concentrations in Fig. 4.

\section{Effects of GDE dosage on mechanical properties and electrical conductivity}

The mechanical strength of the composite hydrogels with CMC concentrations fixed at $7 \%$ and various dosages of GDE are exhibited in Fig. 6. The compressive fracture stress of the composite hydrogels increased at first and then decreased with an increasing dosage of GDE. It had the largest fracture stress $(44.8 \mathrm{kPa})$ when the mole ratio of GDE and AGU was $5: 8$. The compressive strength of the hydrogels was derived from physical and chemical effects of the cross-linked CMC and PAn molecules. There was more cross-linked network density of CMC when the dosage of GDE increased, and the CMC network in the unit volume increased so that the contraction stress of composite hydrogels was enhanced. Modulus of composite hydrogels were also influenced by crosslinker dosages because more crosslinkers make the hydrogel chains highly crosslink to each other which resulted in higher modulus. ${ }^{29}$ The effects of

Table 1 Modulus and conductivity of CMC/PAn composite hydrogels $\left(n_{\mathrm{GDE}}: n_{\mathrm{AGU}}=1: 2\right)$

\begin{tabular}{lcc}
$\begin{array}{l}\text { CMC concentration } \\
(\mathrm{w} / \mathrm{w} \%)\end{array}$ & Modulus $(\mathrm{Pa})$ & $\begin{array}{l}\text { Electrical conductivity } \\
\left(\times 10^{-4} \mathrm{~S} \mathrm{~cm}^{-1}\right)\end{array}$ \\
\hline 5 & $198 \pm 3$ & $0.762 \pm 0.038$ \\
5.5 & $210 \pm 2$ & $2.62 \pm 0.11$ \\
6 & $228 \pm 3$ & $7.73 \pm 0.37$ \\
6.5 & $438 \pm 3$ & $7.52 \pm 0.42$ \\
7 & $467 \pm 5$ & $7.41 \pm 0.36$
\end{tabular}




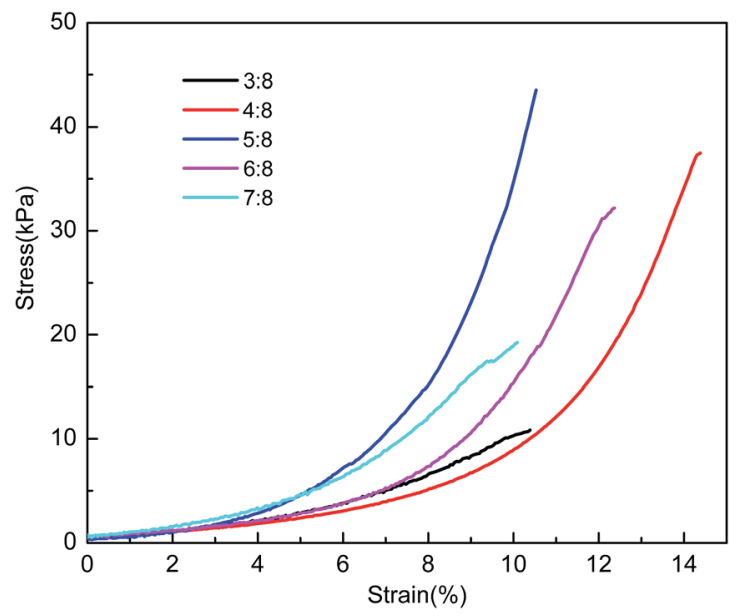

Fig. 6 Compressive stress-strain curves of CMC/PAn composite hydrogels with various dosages of GDE (CMC conc. $=7 \%)$.

GDE dosages on the modulus of CMC/PAn composite hydrogels were shown in Table 2. The modulus of the composite hydrogels increased with more GDE addition. However, the increase of the modulus may enhance the stiffness of the hydrogel which reduces the deformation flexibility of the hydrogel. ${ }^{30}$ It is hard for the compact and rigid composite hydrogels to withstand the increased compressing force by elastic deformation so that they broke down at lower stress for the decreased elastic behaviour with further increasing the dosage of GDE after the ratio of $5: 8$ (Fig. 6).

Effects of GDE dosage on electrical conductivities of the composite hydrogels are demonstrated in Table 2. The dosage of GDE had a great influence on the conductivity of the CMC/ PAn composite hydrogel; the largest conductivity was $7.41 \times$ $10^{-4} \mathrm{~S} \mathrm{~cm}^{-1}$ as the mole ratio of GDE and AGU was $1: 2$. When the cross-linking degree of the composite hydrogels was high, the pore space in the network became smaller; this was disadvantageous for forming an interpenetrating network structure of CMC and PAn, and it led to a decrease in the conductivity of the material. Meanwhile, the high modulus means that the hydrogel chains highly crosslink. The equilibrium swelling ratio tends to decrease with increasing modulus because the hydrogel chains are enriched, and the free volume in the network reduces and thus restrained the expandability of the network $^{29,31}$ and absorption of PAn in the composite hydrogels.

Table 2 Modulus and conductivity of CMC/PAn composite hydrogels at various GDE dosages (CMC conc. $=7 \%$ )

\begin{tabular}{llll}
\hline$n_{\mathrm{GDE}}: n_{\mathrm{AGU}}$ & $\begin{array}{l}\mathrm{BSNa} \\
\left(\mathrm{mol} \mathrm{L}^{-1}\right)\end{array}$ & $\begin{array}{l}\text { Modulus } \\
(\mathrm{Pa})\end{array}$ & $\begin{array}{l}\text { Electrical conductivity } \\
\left(\times 10^{-4} \mathrm{~S} \mathrm{~cm}^{-1}\right)\end{array}$ \\
\hline $3: 8$ & 0 & $452 \pm 6$ & $3.63 \pm 0.19$ \\
$4: 8$ & 0 & $467 \pm 5$ & $7.41 \pm 0.22$ \\
$5: 8$ & 0 & $562 \pm 8$ & $6.8 \pm 0.27$ \\
$6: 8$ & 0 & $620 \pm 8$ & $4.13 \pm 0.21$ \\
$7: 8$ & 0 & $718 \pm 12$ & $3.56 \pm 0.15$ \\
$5: 8$ & 0.5 & - & $63.1 \pm 1.26$
\end{tabular}

Therefore, the electrical conductivity of the composite hydrogels at too high GDE dosages was decreased.

The properties of PAn and the level of electrical conductivity of the polymer depend on the synthesis conditions to a large extent. ${ }^{32}$ PAn doping with a strong acid protonates both iminic nitrogens to give emeraldine salt which is electrically conducting by virtue of its half-filled polaron band. ${ }^{33}$ Normally, bulky sulfonic acids such as aromatic sulfonate are used as dopants to $\mathrm{PAn}^{34}$ to obtain high electrical conductivity and solubility. In this work, BSNa was used to dope PAn in the composite hydrogel in order to increase the conductivity. As biomedical materials and other applications, the conductive hydrogels must possess the sufficient mechanical strength. Therefore, the CMC/PAn composite hydrogel with the largest compressive fracture stress when the mole ratio of GDE and AGU was $5: 8$ was doped with BSNa in order to obtain the composite hydrogel with excellent mechanical and conductive properties. After doping with $0.5 \mathrm{M}$ BSNa, the electrical conductivity of the composite hydrogel reached over $6.31 \times 10^{-3} \mathrm{~S} \mathrm{~cm}^{-1}$, which is much higher than the $1.6 \times 10^{-3} \mathrm{~S} \mathrm{~cm}^{-1}$ of similar conductive hydrogels prepared using a dopant of $\mathrm{HCl}^{22,27}$ It was the dipole and soliton on the PAn chains that improved the conductivity of the composite hydrogel. The benzene sulfonic acid ions were larger than $\mathrm{Cl}^{-}$, which made the PAn chains stretch to form larger chain spacing when BSNa interacted with the main chains of the PAn. The intramolecular and intermolecular conformation of PAn was more conductive to charge delocalization, ${ }^{35}$ thus improving the electrical conductivity of the composite hydrogel.

\section{Experimental section}

\section{Materials}

Carboxymethyl cellulose (CMC) (viscosity: 1000-1400 mPa s, USP grade) was received from Aladdin. GDE was purchased from Sigma-Aldrich. $\mathrm{NaOH}(\geqq 96 \%)$, dimethyl sulfoxide (DMSO), aniline (An) ( $\geq 99.5 \%), \mathrm{HCl}(36-38 \%)$, and ammonium persulfate (APS) $(\geq 98 \%)$ were of analytical grade. Sodium benzene sulfonate (BSNa) was obtained from Shanghai source biological technology Co., Ltd. Distilled water was used throughout this work.

\section{Preparation of CMC/PAn hydrogels}

Conductive composite hydrogels were prepared according to the following procedures: a certain amount of CMC was dissolved in $0.4 \mathrm{M} \mathrm{NaOH}$ solution by continuous mechanical stirring at $80{ }^{\circ} \mathrm{C}$ for 12 hours to obtain homogeneous solution. After cooling to ambient temperature, a certain amount of GDE $(15 \%$ $\mathrm{w} / \mathrm{w}$ in DMSO) was added drop-wise with continuous stirring for 1 hour until a homogeneous mixed solution was obtained. The mixed solution was placed into a water bath at $40{ }^{\circ} \mathrm{C}$ for 24 hours to complete the crosslinking reaction. The resulted CMC hydrogels were washed for at least 72 hours with distilled water to remove impurities. Next, the resulted hydrogels were soaked in $50 \mathrm{~mL}$ of a solution containing aniline $(0.15 \mathrm{M})$ and $\mathrm{BSNa}$ (0 or $0.5 \mathrm{M}$ ) at pH 6.5 for 24 hours; after that, the hydrogels 
were wiped with absorbent paper. Then the hydrogels were immersed in $0.5 \mathrm{M}$ APS solution $(50 \mathrm{~mL})$ at $0{ }^{\circ} \mathrm{C}$ for 6 hours. Lastly, CMC/PAn conductive composite hydrogels were obtained after freeze-drying.

\section{Characterization and analysis}

The molecular structures of CMC/PAn hydrogels were analyzed by nuclear magnetic resonance spectrometer $\left({ }^{13} \mathrm{C}-\mathrm{NMR}\right.$, Bruker plus-400, Germany) and infrared spectroscopy (FT-IR, Tensor27, Bruker, Germany). The morphology of CMC/PAn hydrogels were observed by scanning electron microscope (SEM, S-3700N, Hitachi, Japan).

\section{Swelling behavior}

The swelling ratios (SR) of the hydrogels were calculated according to the formula:

$$
\mathrm{SR}=\left(W_{\mathrm{h}}-W_{\mathrm{d}}\right) / W_{\mathrm{d}}
$$

where $W_{\mathrm{d}}$ is the mass of freeze-dried hydrogels and $W_{\mathrm{h}}$ is the mass of hydrogels after swelling. The swelling experiment was repeated three times and the average value was taken in this work.

\section{Mechanical properties and conductivity}

The mechanical properties of the composite hydrogels were measured via a tensile compression testing machine (Instron5565, USA). Hydrogels were made into cylinders of $15 \mathrm{~mm}$ (diameter) $\times 5 \mathrm{~mm}$ (height) with a hole-punch. The hydrogels were compressed until mechanical failure and the compressive modulus was calculated as the slope of the linear portion of the stress-strain curve. The compressive modulus were calculated as the slope of the linear portion of the stress-strain curve ${ }^{36}$ when the strain was below $8 \%$. The electrical conductivities were measured with a four probe resistance meter (KDY-1, Kunde Science CO., China).

\section{Conclusions}

A non-toxic crosslinking agent was used to prepare CMC/PAn conductive composite hydrogels. The optimum conditions for preparation of the composite hydrogel with the highest strength property were as follows: CMC concentration was $7 \%, n_{\mathrm{GDE}^{-}}$ $: n_{\mathrm{AGU}}=5: 8$. Under these conditions, the compressive fracture stress of the composite hydrogel was $44.8 \mathrm{kPa}$. The maximum swelling ratio of the composite hydrogels was up to 104.3 when the CMC concentration was $5 \%$ and $n_{\mathrm{GDE}}: n_{\mathrm{AGU}}=1: 2$. Both compressive fracture stress and modulus of the composite hydrogels increased as the CMC content increased. Though the modulus of the composite hydrogels increased with more GDE addition, the compressive fracture stress was decreased when the dosage of GDE after the ratio of $5: 8$ because of the decreased elastic behaviour. The electrical conductivity of the composite hydrogels varied with the concentration of CMC and the amount of crosslinking agent, and the conductivity increased first and then decreased slightly with increasing dosages because the condensed network restrained the absorption of PAn in the composite hydrogels. After doping with BSNa, the electrical conductivity of the composite hydrogel could reach $6.31 \times 10^{-3} \mathrm{~S} \mathrm{~cm}^{-1}$. All of the results showed that the CMC/PAn composite hydrogels have potential for applications in electro-stimulated controlled drug release, sensing materials and other fields.

\section{Conflicts of interest}

There are no conflicts to declare.

\section{Acknowledgements}

The authors are grateful for the support of the grants from the Guangdong Science and Technology Project of China (2014A010105012).

\section{Notes and references}

1 S. Kakuda, B. T. Momma and C. T. Osaka, J. Electrochem. Soc., 1995, 142, 1.

2 J. A. Killion, L. M. Geever, D. M. Deuine, J. E. Kennedy and C. L. Higginbotham, J. Mech. Behav. Biomed. Mater., 2011, 4, 1219.

3 J. B. Prettyman and D. T. Eddington, Sens. Actuators, B, 2011, 157, 722.

4 B. L. Guo and Q. Y. Gao, Carbohydr. Res., 2007, 342, 2416.

5 S. Miyazaki, T. Karino, H. Endo, K. Haraguchi and M. Shibayama, Macromolecules, 2006, 39, 8112.

6 A. Khoushabi, A. Schmocker, D. P. Pioletti, C. Moser, C. Schizas, J. A. Månson and P. E. Bourban, Compos. Sci. Technol., 2015, 119, 93.

7 N. Astrini, L. Anah and H. R. Haryadi, Macromol. Symp., 2015, 353, 191.

8 L. Marija, M. Nedeljko, R. Maja, Š. Zoran, R. Marija and K. K. Melina, Polym. Compos., 2014, 35, 806.

9 R. Bhattacharyya and S. K. Ray, Ind. Eng. Chem., 2015, 22, 92.

10 E. A. Kamoun, E. S. Kenawy, T. M. Tamer, M. A. El-Meligy and M. S. MohyEldin, Arabian J. Chem., 2015, 8, 38.

11 K. Baysal, A. Z. Aroguz, Z. Adiguzel and B. M. Baysal, Int. J. Biol. Macromol., 2013, 59, 342.

12 M. C. Li, C. T. Mei, X. W. Xu, S. Lee and Q. L. Wu, Polymer, 2016, 107, 200.

13 M. Hashem, S. Sharaf, M. M. Abd El-Hady and A. Hebeish, Carbohydr. Polym., 2013, 95, 421.

14 G. Teti, V. Salvatore, S. Focaroli, S. Durante, A. Mazzotti, M. Dicarlo, M. M. Belmonte and G. Orsini, Front. Physiol., 2015, 6, 297.

15 A. Salama, Mater. Lett., 2015, 157, 243.

16 A. Salama, N. Shukry and M. El-Sakhawy, Int. J. Biol. Macromol., 2015, 73, 72.

17 M. Yadollahia, I. Gholamalia, H. Namazia and M. Aghazadeh, Int. J. Biol. Macromol., 2015, 74, 136.

18 X. Gong, C. Branford-White, L. Tao, S. B. Li, J. Quan, H. L. Nie and L. M. Zhu, Mater. Sci. Eng., C, 2016, 58, 478. 19 I. Jun, S. Jeong and H. Shin, Biomaterials, 2009, 30, 2038. 
20 A. Lewandowski, M. Zajder, E. Frackowiak and F. Beguin, Electrochim. Acta, 2001, 46, 2777.

21 P. Pissis and A. Kyritsis, Solid State Ionics, 1997, 97, 105.

22 V. Guarino, M. A. Alvarez-Perez, A. Borriello, T. Napolitan and L. Ambrosio, Adv. Healthcare Mater., 2013, 2, 218.

23 X. Zhao, P. Li, B. L. Guo and P. X. Ma, Acta Biomater., 2015, 26, 236.

24 P. C. Du, H. C. Liu, C. Yi, K. Wang and X. Gong, ACS Appl. Mater. Interfaces, 2015, 7, 23932.

25 K. Sharma, B. S. Kaith, V. Kumar, S. Kalia, V. Kumar and H. C. Swart, Geoderma, 2014, 232, 45.

26 A. I. Ramos, T. M. Braga, J. A. Fernandes, P. Silva, P. J. Ribeiro-Claro, F. A. A. Paz, M. F. S. Lopes and S. S. Braga, J. Pharm. Biomed. Anal., 2013, 80, 34.

27 L. Zhang, Y. Li, L. C. Li, B. L. Guo and P. X. Ma, React. Funct. Polym., 2014, 82, 81.
28 S. Sheth, E. Jain, A. Karadaghy, S. Syed, H. Stevenson and S. P. Zustiak, Int. J. Polym. Sci., 2017, 5147482.

29 H. Li and R. M. Luo, Biosens. Bioelectron., 2009, 24, 3630.

30 A. Srivastava, E. Jain and A. Kumar, Mater. Sci. Eng., A, 2007, 464, 93.

31 F. K. Lai, H. Li and R. M. Luo, Int. J. Solids Struct., 2010, 47, 141.

32 I. Sapurina, A. V. Tenkovtsev and J. Stejskal, Polym. Int., 2015, 64, 453.

33 J. P. Foreman and A. P. Monkman, J. Phys. Chem. A, 2003, 107, 7604.

34 S. M. Ahmed and S. A. Ahmed, Eur. Polym. J., 2002, 38, 25.

35 J. P. Foreman and A. P. Monkman, J. Phys. Chem. A, 2003, 107, 7604-7610.

36 E. C. Beck, M. Barragan, M. H. Tadros, S. H. Gehrke and M. S. Detamore, Acta Biomater., 2016, 38, 94. 